\title{
Comparison of Endoscopic Ultrasound-Guided Fine-Needle Aspiration and Biopsy Device for Lymphadenopathy
}

\author{
Yuki Tanisaka $\mathbb{D}^{1},{ }^{1}$ Masafumi Mizuide, ${ }^{1}$ Akashi Fujita, ${ }^{1}$ Tomoya Ogawa, ${ }^{1}$ Ryuichiro Araki, ${ }^{2}$ \\ Masahiro Suzuki, ${ }^{1}$ Hiromune Katsuda, ${ }^{1}$ Youichi Saito, ${ }^{1}$ Kazuya Miyaguchi, ${ }^{1}$ \\ Tomoaki Tashima, ${ }^{1}$ Yumi Mashimo, ${ }^{1}$ Masami Yasuda, ${ }^{3}$ and Shomei Ryozawa ${ }^{1}$ \\ ${ }^{1}$ Department of Gastroenterology, Saitama Medical University International Medical Center, Japan \\ ${ }^{2}$ Saitama Medical University, Community Health Science Center, Japan \\ ${ }^{3}$ Department of Pathology, Saitama Medical University International Medical Center, Japan \\ Correspondence should be addressed to Yuki Tanisaka; tanisaka1205@gmail.com
}

Received 2 March 2021; Revised 5 April 2021; Accepted 8 April 2021; Published 15 April 2021

Academic Editor: Konstantinos Triantafyllou

Copyright (C) 2021 Yuki Tanisaka et al. This is an open access article distributed under the Creative Commons Attribution License, which permits unrestricted use, distribution, and reproduction in any medium, provided the original work is properly cited.

\begin{abstract}
Background. Accurate diagnosis of benign and malignant lymphadenopathy is important for determining the appropriate treatment and prognosis. This study evaluated the diagnostic accuracy and usefulness of endoscopic ultrasound-guided fineneedle aspiration (EUS-FNA) with a conventional needle compared to endoscopic ultrasound-guided fine-needle biopsy (EUSFNB) with a Franseen needle for diagnosing lymphadenopathy. Methods. Patients who underwent EUS-FNA or EUS-FNB for mediastinal or abdominal lymphadenopathy between July 2013 and August 2020 were enrolled in the study. The outcomes between EUS-FNA patients (July 2013 to January 2017; 22-gauge conventional needle; Group A) and EUS-FNB patients (February 2017 to August 2020; 22-gauge Franseen needle; Group B) were compared. Results. A total of 154 patients (Group A: 83; Group B: 71) were analyzed. The diagnostic accuracy (differentiating between malignant and benign lesions) was $88.0 \%$ (95\% confidence interval [CI], 79.2-93.3\%) in Group A and 95.8\% (95\% CI, 88.3-98.8\%) in Group B. Group B had high diagnostic accuracy, but there was no difference between the groups $(p=0.14)$. Group B had significantly fewer passes (median 2, interquartile range (IQR): 2-4) than Group A (median 3, IQR: 3-4) $(p<0.001)$. No procedural adverse events occurred in either group. Conclusions. Although the diagnostic accuracy between the groups was not statistically significant, EUS-FNB with a Franseen needle provided high diagnostic accuracy and required fewer passes to establish a diagnosis. Thus, EUS-FNB is useful for diagnosing lymphadenopathy.
\end{abstract}

\section{Introduction}

Mediastinal and abdominal lymphadenopathy present with numerous symptoms, and accurate diagnosis is important to determine the appropriate treatment and prognosis [1]. Although cross-sectional imaging, such as computed tomography, magnetic resonance imaging, and positron emission tomography, is useful for detecting lymphadenopathy, it is difficult to distinguish between benign and malignant lesions using only imaging modalities [2-4]. Invasive procedures, such as open thoracic surgery, thoracoscopy, and laparoscopy, were previously required for histological diagnosis.
Endoscopic ultrasound (EUS) can easily access the lymph nodes and provide detailed information on the shape, diameter, and internal echoic features via high-resolution images [5-7]. The features of malignant lymph nodes reported on EUS images are a diameter of $10 \mathrm{~mm}$ or greater, round shape, sharply demarcated borders, and a homogeneous and hypoechoic central echo pattern. EUS-guided fine-needle aspiration (EUS-FNA) has been used for the diagnosis of many lesions since it was first reported in 1992 [8]. EUS-FNA is a minimally invasive method for collecting diagnostic cytological and histological materials for lymphadenopathy compared to surgery. Moreover, it can be used to determine 
cancer stages and the etiology and surgically resect malignancy recurrences. Therefore, EUS-FNA is useful for the lymphadenopathy diagnostic process [9-16]. A recent systematic review and meta-analysis reported that the pooled sensitivity and specificity were $87 \%$ and $100 \%$, for differentiating benign and malignant lymphadenopathy, respectively [17]. However, fine-needle aspiration (FNA) tissue sampling sometimes cannot provide enough material for diagnosis.

With the recent progress of needles, the fine-needle biopsy (FNB) device, designed primarily to obtain core tissue samples, was introduced to overcome the FNA sampling material limitation $[18,19]$. The usefulness of the FNB needle has been extensively reported $[20,21]$. Immunostaining is often required for lymphadenopathy diagnosis and requires a large tissue sample, which can be provided by the FNB needle. The Franseen needle, a type of FNB needle used in our facility, has a crown tip with three symmetrical surfaces that manifest as three cutting edges. This unique design is expected to obtain adequate tissue amounts. Moreover, the needle is made of cobalt-chromium, a highly durable alloy; this allows repeat punctures without needle dysfunction. Previous reports have shown that large tissue sample amounts were obtained using a Franseen needle [19, 22].

Previous studies have compared the utility of conventional and FNB needles for diagnosing lymphadenopathy in a small number of cases [23-25]. Therefore, this study was aimed to compare the diagnostic accuracy and usefulness between EUS-FNA with a conventional needle and EUS-FNB with a Franseen needle for diagnosing lymphadenopathy.

\section{Methods}

This is a retrospective, single-center study approved by the Institutional Review Board at Saitama Medical University International Medical Center (18-253). All patients provided written informed consent before undergoing the procedure.

2.1. Patients. We planned to perform EUS-FNA or EUS-FNB in patients with mediastinal or abdominal lymphadenopathy that could not be diagnosed using only cross-sectional imaging at our facility between July 2013 and August 2020. The inclusion criteria were as follows: lymphadenopathy $>10 \mathrm{~mm}$ in maximal diameter; and lymphadenopathy indicated using cross-sectional imaging to be approachable with EUS-FNA or EUS-FNB from the esophagus, stomach, or duodenum. The exclusion criteria were as follows: superficial lymphadenopathy that could permit a superficial approach to obtain tissue; patients treated with antithrombotic agents that may not be discontinued; lymphadenopathy performed via EUS-FNA or EUS-FNB using a 25-gauge needle (Expect ${ }^{\circledR}$, Boston Scientific, Marlborough, MA, USA). Patients were divided into two groups: Group A for EUS-FNA (July 2013 to January 2017; 22-gauge conventional needle) and Group B for EUS-FNB (February 2017 to August 2020; 22-gauge Franseen needle).

2.2. Procedures. EUS procedures were performed by four training fellows with experience in less than 50 EUS-FNA procedures under the direct supervision of endoscopists with experience in over 100 EUS-FNA procedures. In cases where the training fellows encountered difficulties in completing the procedure, the experienced endoscopists completed it. Two training fellows performed procedures in each group. They did not perform procedures across both groups. All procedures were performed with the patient under conscious sedation via a combination of intravenous midazolam and pethidine.

The EUS-FNA and EUS-FNB procedures used a linear echoendoscope (GF-UCT260; Olympus Medical Systems, Tokyo, Japan) paired with an ultrasound system (EU-ME2 Premier Plus; Olympus Medical Systems, Tokyo, Japan). The swollen lymph node was visualized, the regional vasculature was excluded using the color Doppler mode, and then, the target lesion was punctured. Next, the stylet was removed, and continuous suction was applied using a $20 \mathrm{~mL}$ syringe. After 20 rapid suction strokes within the lesion, the suction was released, and the needle was removed. The obtained tissue specimens were pushed out by reinserting the stylet or creating positive pressure using air.

Rapid on-site evaluation (ROSE) is not available at our facility. Thus, the sample was examined macroscopically to determine whether the specimen was of a sufficient quantity. We also checked the color of specimens, because a red specimen was thought to be blood while a white specimen was thought to be tissue. These examinations were performed under the supervision of a pathology technician. When a certain amount of specimen (including a certain proportion of white specimen) was obtained, we finished the procedure. If the macroscopic results showed an increase in blood components, the negative pressure level of the syringe was decreased or no suction was applied, as necessary.

The Expect $^{\circledR}$ conventional needle (Boston Scientific, Marlborough, MA, USA) was used for Group A, and the Acquire ${ }^{\circledR}$ Franseen needle (Boston Scientific, Marlborough, MA, USA) was used for Group B (Figure 1).

2.3. Sampling Evaluation. Smears were alcohol-fixed (95\% ethanol, room temperature for $15 \mathrm{~min}$ ), and subsequently, Papanicolau stained in the laboratory for cytological examination. The sample was checked for adequacy and then preserved in $10 \%$ neutral buffered formalin, embedded in paraffin, and cut into $4 \mu \mathrm{m}$ thick serial sections for hematoxylin and eosin staining for histological examination. Immunostaining was performed if needed. Two pathology technicians and two pathologists examined the sections.

2.4. Definitions and Outcome Measurement. Patients' age, sex, lymph node diameter, lymph node location, puncture site, number of passes, procedure time, and final diagnosis were recorded from electronic medical records. The final diagnosis was classified as malignant or benign lymph nodes, and the sensitivity, specificity, positive predictive value, negative predictive value, and accuracy for differentiating between malignant and benign lesions were estimated. The final diagnosis was confirmed through histological evaluation of the resected specimen when surgery was performed. For patients who did not undergo surgery, the final diagnosis 


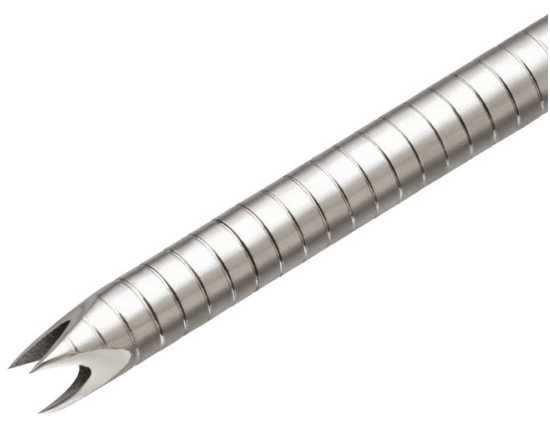

(a)

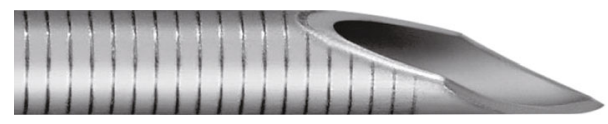

(b)

Figure 1: Endoscopic ultrasound-guided fine needles. (a) A Franseen needle with three symmetric cutting tips for endoscopic ultrasoundguided fine-needle biopsy (EUS-FNB). (b) A conventional needle for endoscopic ultrasound-guided fine-needle aspiration (EUS-FNA).

was defined as malignant when histological evaluation of EUS-FNA- or EUS-FNB-based tissue confirmed malignancy and the lymph node became larger on follow-up imaging evaluations. In patients without histological confirmation, the final diagnosis was defined as malignant when the clinical course or cross-sectional imaging evaluations consistently worsened during the follow-up period. The final diagnosis was defined as benign if malignant findings were absent on histological examinations and there was no progression after more than six months of follow-up. Technical success was defined as EUS-FNA or EUS-FNB completion with a successful acquisition of macroscopically visible whitish material.

2.5. Statistical Analysis. The Shapiro-Wilk's W test showed that all continuous variables were not normally distributed. Thereby, these variables were expressed as medians and interquartile ranges (IQR), and the Mann-Whitney $U$ test was used to compare continuous data. Between-group categorical data comparisons were performed using Fisher's exact test to calculate 2 -tailed $p$ values; $p<0.05$ was considered statistically significant. Statistical data were calculated using SAS JMP (version 14.2.0; SAS Institute Inc., Cary, NC, USA).

\section{Results}

3.1. Patients. Figure 2 provides a flowchart of patients' selection. In the EUS-FNA group (July 2013 to January 2017), 96 patients were initially included. We excluded 3 patients with superficial lymphadenopathy, 5 patients with antithrombotic therapy, and 5 patients who underwent EUS-FNA using a 25 -gauge needle. Finally, 83 patients were included in the EUS-FNA group (Group A).

In the EUS-FNB group (February 2017 to August 2020), 81 patients were initially included. We excluded 3 patients with superficial lymphadenopathy, 4 patients with antithrombotic therapy, and 3 patients who underwent EUSFNB using a 25-gauge needle. Finally, 71 patients were included in the EUS-FNB group (Group B). A total of 154 patients (Group A: 83; Group B: 71) were analyzed. The patient characteristics of Group A and Group B are presented in Table 1. Age, sex, lymph node diameter, and lymph node location did not significantly differ between the groups. A 25 -gauge needle was used when the lymphadenopathy was small (within $15 \mathrm{~mm}$ ) and located near the vessel.

3.2. Final Diagnoses. The final diagnoses are presented in Table 2. A total of 138 patients had malignant lesions (metastatic, 88 (Group A: 42, Group B: 46); malignant lymphoma, 50 (Group A: 33, Group B: 17)), and 16 had benign lesions (reactive change, 15 (Group A: 8, Group B: 7); sarcoidosis, 1 (Group A: 1)). The diagnosis that was obtained through surgery was in 3 cases. The lymphadenopathy was located in the para-aortic region in one case. Although lymphoma was the chief probable diagnosis, the results of EUS-FNA showed no malignancy. Finally, open surgical resection from the lymphadenopathy was performed. The final diagnosis was T-cell lymphoma. In 2 cases, the lymphadenopathy was located near pancreatic cancer and duodenal cancer lesions. It was difficult to obtain sufficient samples from the main lesions to make a correct diagnosis. Therefore, EUS-FNB was performed in the lymphadenopathy near the main lesions. Finally, the main lesions were resected, including the lymphadenopathy, and they were diagnosed as adenocarcinoma. However, we must be mindful that it is difficult to confirm whether the lymph node resected and evaluated to establish the final diagnosis was the one we sampled during EUS.

We evaluated EUS findings [5-7]. As the diameter was $10 \mathrm{~mm}$ or greater in all cases, we evaluated whether sharply demarcated borders, round shape, and homogeneous and hypoechoic central echo pattern were present. Sharply demarcated borders, round shape, and homogeneous and hypoechoic central echo pattern were found in $45.7 \%$, $49.3 \%$, and $61.6 \%$ of malignant lesions and in $37.5 \%, 18.8 \%$, and $56.3 \%$ of benign lesions (Table 3), respectively. Although assessment of EUS findings was useful, it was difficult to reach a correct diagnosis without EUS-FNA or EUS-FNB.

\subsection{Procedure Outcomes between Conventional and Franseen} Needle Groups. Procedural outcome comparisons between the conventional (Group A) and Franseen (Group B) needle groups are presented in Table 4 . Group B had significantly fewer passes (median 2, IQR: 2-4) than Group A (median 3, IQR: $3-4)(p<0.001)$. There were no differences in procedure 


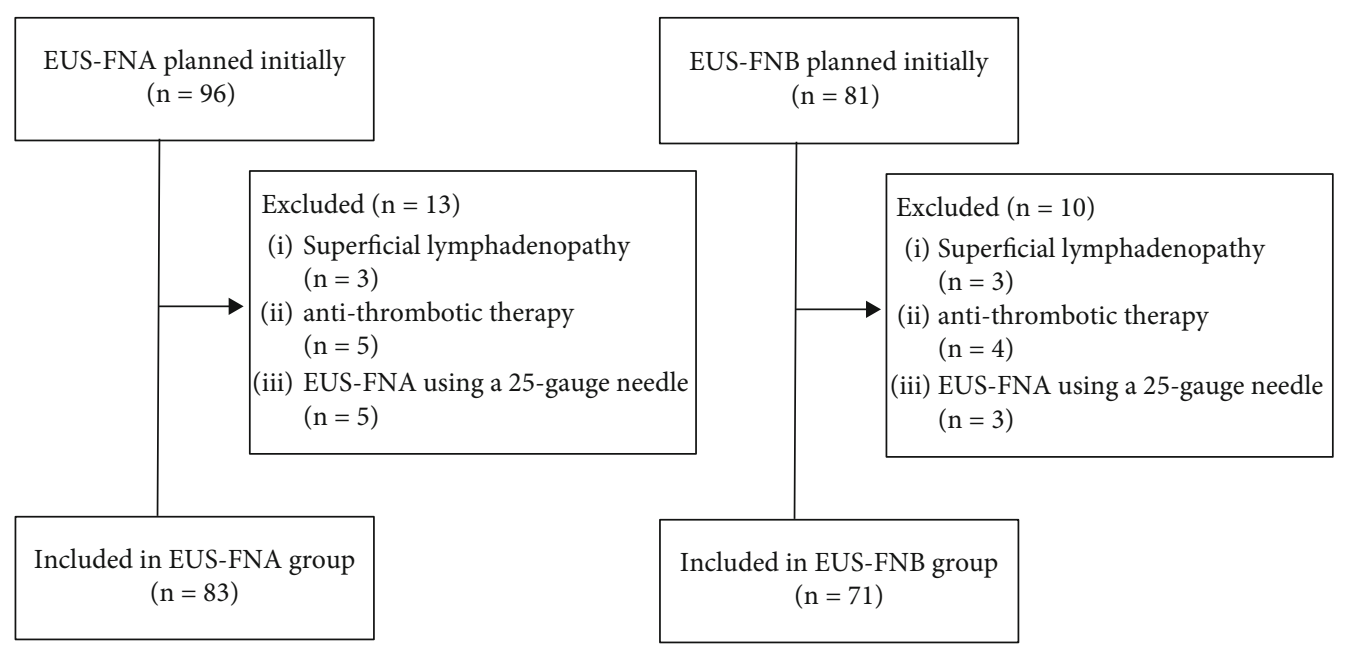

FIGURE 2: A flowchart of patients' selection between EUS-FNA and EUS-FNB groups.

TABLE 1: Patient characteristics between conventional (Group A) and Franseen (Group B) needle groups.

\begin{tabular}{|c|c|c|c|}
\hline & Group A & Group B & $p$ value \\
\hline Patients, $n(\%)$ & 83 & 71 & \\
\hline Age, median (IQR), years & $67(60-74)$ & $70(61-74)$ & 0.38 \\
\hline Gender (male), $n(\%)$ & $51(61.4)$ & $36(50.7)$ & 0.20 \\
\hline Diameter of lymph node, median (IQR), mm & $22(15-35)$ & $26(19-35)$ & 0.32 \\
\hline Location of lymph node, $n(\%)$ & & & $>0.99$ \\
\hline Abdominal lymphadenopathy & $77(92.8)$ & $65(91.5)$ & \\
\hline Mediastinum lymphadenopathy & $6(7.2)$ & $6(8.5)$ & \\
\hline
\end{tabular}

$n$ : number of cases; IQR: interquartile range.

TABLE 2: Final lymphadenopathy diagnoses $(n=154)$.

\begin{tabular}{lc}
\hline Final diagnosis & $n$ (\%) \\
\hline Total of malignant lesions & 138 (89.6) (Group A: 75, Group B: 63) \\
Metastasis & $88(57.1)$ (Group A: 42, Group B: 46) \\
Malignant lymphoma & $50(32.5)$ (Group A: 33, Group B: 17) \\
Total of benign lesions & $16(10.4)$ (Group A: 8, Group B: 8) \\
Reactive change & $15(9.7)$ (Group A: 8, Group B: 7) \\
Sarcoidosis & $1(0.7)$ (Group A: 1) \\
\hline
\end{tabular}

Group A: conventional needle group Group B: Franseen needle group. $n$ : number of cases.

time, and the technical success rate was $100 \%$ in both groups. No procedural adverse events occurred in either group. We also performed a subgroup analysis regarding malignant lymphoma. Group B had significantly fewer passes (median 3, IQR: 3-4) than Group A (median 4, IQR: 4-5) $(p<0.001$ ). There were no differences in procedure time (Table 5).

3.4. Histological Diagnostic Accuracy between Conventional and Franseen Needle Groups. Histological diagnostic accuracy comparisons between the conventional (Group A) and Franseen (Group B) needle groups are presented in Table 6. Diagnostic accuracy did not differ according to the puncture route, tumor type, and tumor size. The diagnostic accuracy from transduodenal puncture was higher in Group B
(92.3\%) than in Group A (69.2\%), but this difference was not statistically significant $(p=0.15)$. Moreover, the diagnostic accuracy of metastasis in Group B (93.5\%) was higher than that in Group A $(81.0 \%)$, but this difference was also not statistically significant $(p=0.11)$. We also performed a subgroup analysis regarding malignant lymphoma. Diagnostic accuracy did not differ according to the puncture route and tumor size (Table 5).

3.5. Diagnostic Performance for Differentiating Malignant and Benign Lesions. Histological diagnostic performance comparisons for differentiating between malignant and benign lesions are presented in Table 7. The results were as follows: sensitivity (Group A: 86.7\% [65/75]; Group B: 95.2\% [60/63]), specificity (Group A: 100\% [8/8]; Group B: $100 \%$ [8/8]), positive predictive value (Group A: $100 \%$ [65/65]; Group B: $100 \%$ [60/60]), negative predictive value (Group A: 44.4\% [8/18]; Group B: 72.7\% [8/11]), and accuracy (Group A: 88.0\% [73/83], 95\% confidence interval [CI], 79.2-93.3\%; Group B: 95.8\% [68/71], 95\% CI, 88.3$98.8 \%)$. Group B had a higher diagnostic accuracy, but there was not significantly different $(p=0.14)$. We also analyzed the diagnostic accuracy using cytology. It was $83.1 \%(95 \%$ CI, 73.7-89.7\%) for EUS-FNA (Group A) and 91.5\% (95\% CI, 82.8-96.1\%) for EUS-FNB (Group B). Group B had high diagnostic accuracy, but there was no difference between the groups $(p=0.15)$. Moreover, combined diagnostic accuracy 
TABLE 3: EUS findings on malignant and benign lymphadenopathy.

\begin{tabular}{lccc}
\hline & Sharply demarcated border & Rounded shape & Homogeneous and hypoechoic central echo pattern \\
\hline Malignant lesions, $n(\%)$ & $63 / 138(45.7)$ & $68 / 138(49.3)$ & $85 / 138(61.6)$ \\
Benign lesions, $n(\%)$ & $6 / 16(37.5)$ & $3 / 16(18.8)$ & $9 / 16(56.3)$ \\
\hline
\end{tabular}

$n$ : number of cases.

TABLE 4: Procedural comparisons between the conventional (Group A) and Franseen (Group B) needle groups.

\begin{tabular}{lccc}
\hline & Group A & Group B & $p$ value \\
\hline Number of punctures, median (IQR) & $3(3-4)$ & $2(2-4)$ & $<0.001$ \\
Procedure time (min), median (IQR) & $27(20-32)$ & $26(23-29)$ & 0.32 \\
Technical success, $n(\%)$ & $83 / 83(100)$ & $71 / 71(100)$ & $>0.99$ \\
Adverse events, $n(\%)$ & $0 / 83(0)$ & $0 / 71(0)$ & $>0.99$ \\
\hline
\end{tabular}

$n$ : number of cases; IQR: interquartile range.

TABLE 5: Comparison of procedure results and diagnostic accuracy between conventional (Group A) needle and Franseen (Group B) needle groups in malignant lymphoma.

\begin{tabular}{lccc}
\hline & Group A & Group B & $p$ value \\
\hline Number of punctures, median (IQR) & $4(4-5)$ & $3(3-4)$ & $<0.001$ \\
Procedure time (min), median (IQR) & $27(22-31)$ & & \\
Diagnostic accuracy, $n$ (\%) & & & \\
Puncture route & & 0.92 \\
$\quad$ Transesophageal & $1 / 1(100)$ & $0 / 0$ & $>(10)$ \\
Transgastric & $25 / 27(92.6)$ & $7 / 7(100)$ & $>0.99$ \\
Transduodenal & $5 / 5(100)$ & $15 / 15(100)$ \\
Tumor size & & $2 / 2(100)$ & $>0.99$ \\
$\geq 20$ mm & $23 / 24(95.8)$ & $17 / 17(100)$ \\
$<20$ mm & $8 / 9(88.9)$ & $>0.99$ \\
Overall & $31 / 33(94.0)$ & \\
\hline
\end{tabular}

$n$ : number of cases; IQR: interquartile range.

TABLE 6: Histological diagnostic accuracy comparisons between the conventional (Group A) and Franseen (Group B) needle groups.

\begin{tabular}{lccc}
\hline Diagnostic accuracy, $n(\%)$ & Group A & Group B & $p$ value \\
\hline Puncture route & & & \\
$\quad$ Transesophageal & $5 / 6(83.3)$ & $6 / 6(100)$ & $>0.99$ \\
Transgastric & $59 / 64(92.2)$ & $38 / 39(97.4)$ & 0.40 \\
$\quad$ Transduodenal & $9 / 13(69.2)$ & $24 / 26(92.3)$ & 0.15 \\
Tumor type & & & \\
Metastasis & $34 / 42(81.0)$ & $43 / 46(93.5)$ & 0.11 \\
Malignant lymphoma & $31 / 33(94.0)$ & $17 / 17(100)$ & 0.54 \\
Benign lesions & $8 / 8(100)$ & $8 / 8(100)$ & $>0.99$ \\
Tumor size & & & \\
$\geq 20$ mm & $49 / 55(89.1)$ & $44 / 46(95.7)$ & 0.29 \\
$\quad<20$ mm & $24 / 28(85.7)$ & $24 / 25(96)$ & 0.35 \\
Overall & $73 / 83(88.0)$ & $68 / 71(95.8)$ & 0.14 \\
\hline
\end{tabular}

$n$ : number of cases.
TABLE 7: Histological diagnostic performance comparisons for differentiating between malignant and benign lesions.

\begin{tabular}{lccc}
\hline $\begin{array}{l}\text { Diagnostic accuracy, } n(\%), 95 \% \\
\text { CI }\end{array}$ & Group A & Group B & $\begin{array}{c}p \\
\text { value }\end{array}$ \\
\hline & $65 / 75$ & $60 / 63$ & \\
Sensitivity & $(86.7)$ & $(95.2)$ & 0.14 \\
& $77.2-92.6$ & $87.0-98.7$ & \\
Specificity & $8 / 8(100)$ & $8 / 8(100)$ & $>0.99$ \\
& $67.6-100$ & $67.6-100$ & \\
Positive predictive value & $65 / 65$ & $60 / 60$ & \\
& $(100)$ & $(100)$ & $>0.99$ \\
& $94.4-100$ & $94.0-100$ & \\
Negative predictive value & $8 / 18(44.4)$ & $8 / 11(72.7)$ & 0.25 \\
& $24.6-66.3$ & $43.4-90.3$ & \\
Accuracy & $73 / 83$ & $68 / 71$ & \\
& $(88.0)$ & $(95.8)$ & 0.14 \\
\hline
\end{tabular}

$n$ : number of cases; CI: confidence interval. 
TABLE 8: Diagnostic accuracy comparisons between the conventional (Group A) and Franseen (Group B) needle groups regarding cytology, histology, and combined cytology and histology.

\begin{tabular}{lccc}
\hline Diagnostic accuracy, $n(\%), 95 \%$ CI & Group A & Group B & $p$ value \\
\hline Cytology & $69 / 83(83.1)$ & $65 / 71(91.5)$ & $82.8-96.1$ \\
& $73.7-89.7$ & $68 / 71(95.8)$ & 0.15 \\
Histology & $73 / 83(88.0)$ & $88.3-98.8$ & 0.14 \\
& $79.2-93.3$ & $68 / 71(95.8)$ & 0.23 \\
Combined cytology and histology & $75 / 83(90.4)$ & $88.3-98.8$ & \\
\hline
\end{tabular}

$n$ : number of cases; CI: confidence interval.

using cytology and histology was $90.4 \%$ (95\% CI, $82.1-$ 95.0\%) in Group A and 95.8\% (95\% CI, 88.3-98.8\%) in Group B. There was no difference between the groups $(p=0.23)$ (Table 8$)$.

\section{Discussion}

Previous reports compared the utility of conventional and FNB needles for diagnosing lymphadenopathy in only a small number of cases [23-25]. Thus, we conducted a comparison study using a larger number. In this study, the diagnostic accuracy for differentiating between malignant and benign lymphadenopathy using a Franseen needle (Group B) was higher than that of the accuracy using a conventional needle (Group A) (95.8\% vs. 88\%), although this difference was not statistically significant. Previous reports stated that the lymphadenopathy diagnostic accuracy using a conventional needle was over $85 \%$ [9-16], making it difficult to show significant differences compared to that obtained using a Franseen needle.

In this study, the Franseen needle showed several benefits. First, the Franseen needle required significantly fewer passes than the conventional needle with no available ROSE, even in cases of malignant lymphoma. Although we did not evaluate the accurate amount of tissue, this result may suggest that larger amounts of tissue could be acquired using a Franseen needle, making it easier to perform the macroscopic identification of tissue samples. Although ROSE improves diagnostic accuracy [26], it is not always available, even in high-volume centers such as ours. In such facilities, the FNB needle facilitates the macroscopic examination of tissue samples. Moreover, fewer passes minimize the risk of trauma to the tissue or vessels. Second, the diagnostic accuracy of metastasis using a Franseen needle was high (although not significantly different between the groups). For indeterminate lymphadenopathy diagnosis (i.e., the cancer etiology cannot be identified by cross-sectional imaging or EUS findings), larger amounts of tissue are useful for the correct diagnosis. More tissue is also beneficial for immunostaining. If the EUS-FNB and surgical specimens are histologically similar, recurrent cancer is diagnosed. Thus, a larger specimen is more useful for cancer diagnosis, and the Franseen needle could provide this. Third, the EUS-FNB technical success rate was $100 \%$ despite the hypothesis that the Franseen needle tip may make puncturing difficult. This was particularly worrisome for transduodenal punctures, but the accurate diagnosis of transduodenal punctures was extremely satisfactory $(92.3 \%)$. The Franseen needle tip may induce more localized trauma, resulting in more frequent adverse events such as bleeding. Fortunately, there was no adverse event using a Franseen needle in this study. However, we must be mindful that adverse events, such as bleeding, can occur with a Franseen needle.

This study is retrospective and was conducted over two distinct periods; EUS-FNA was performed in the first period (in 83 patients) and EUS-FNB in the second (in 71 patients). Therefore, the learning curve must be taken into consideration. All procedures were initially performed by training fellows with less experience under the direct supervision of experienced endoscopists, and training fellows did not perform procedures across both groups. Therefore, we investigated the diagnostic accuracy differences between the beginning and end of each period using EUS-FNA (beginning: 42 cases, end: 41 cases) and EUS-FNB (beginning: 36 cases, end: 35 cases) to determine the influence of the learning curve. The diagnostic accuracies of the EUS-FNA in the beginning and end periods were $90.5 \%$ (38/42) and $85.4 \%$ $(35 / 41)$, respectively, and there was no difference between the periods $(p=0.52)$. The diagnostic accuracies of the EUS-FNB in the beginning and end periods were $97.2 \%$ (35/36) and $94.3 \%(33 / 35)$, respectively, also with no difference between the periods $(p=0.61)$. The learning curve cannot be completely discounted, but it does not have a considerable influence on outcomes.

EUS-FNB allows for a wide range of tests for personalized diagnosis. Moreover, it enables genetic testing, which is useful in the individualized medicine era [27]. Hence, EUSFNB may expand the available diagnosis and treatment tools, even for lymphadenopathy.

The fork-tip needle is another EUS-FNB needle which provides a high rate of histological specimens [28], although we have not used it in this study. This needle is also useful for the diagnosis of lymphadenopathy.

This study has several limitations. First, it was a singlecenter retrospective analysis. Moreover, it was not a sideby-side comparative study, and not randomized. Second, as previously mentioned, the procedure was performed by four endosonographers-in-training, which carries a heterogeneity risk between operators despite having been performed under the direct supervision of experienced endoscopists. A prospective study with a larger number of cases is necessary. Moreover, we used only the continuous suction technique 
using a $20 \mathrm{~mL}$ syringe. Continuous suction with the stylet technique (the stylet slow-pull technique), which has been reported as useful to avoid blood contamination [29-31], would be useful for lymphadenopathy in future studies.

In conclusion, although there was no difference in the diagnostic accuracy between the groups, EUS-FNB with a Franseen needle provided high diagnostic accuracy and required fewer passes to establish a diagnosis. Therefore, EUS-FNB is useful for diagnosing lymphadenopathy.

\section{Data Availability}

The patient data used to support the findings of this study are restricted by the Institutional Review Board at Saitama Medical University International Medical Center in order to protect PATIENT PRIVACY. Data are available from Yuki Tanisaka, tanisaka1205@gmail.com for researchers who meet the criteria for access to confidential data.

\section{Conflicts of Interest}

The authors declare that they have no competing interests.

\section{Authors' Contributions}

The paper was authored by Yuki Tanisaka, who designed and drafted the article. Masafumi Mizuide, Akashi Fujita, Tomoya Ogawa, Ryuichiro Araki, Masahiro Suzuki, Hiromune Katsuda, Youichi Saito, Kazuya Miyaguchi, Tomoaki Tashima, Yumi Mashimo, Masami Yasuda, and Shomei Ryozawa provided critical revision of the article for important intellectual content. Yuki Tanisaka finally approved the article for submission. The final version of the manuscript was approved by all authors.

\section{Acknowledgments}

We would like to thank Editage (http://www.editage.com) for English language editing.

\section{References}

[1] P. Vilmann, P. F. Clementsen, S. Colella et al., "Combined endobronchial and esophageal endosonography for the diagnosis and staging of lung cancer: European Society of Gastrointestinal Endoscopy (ESGE) Guideline, in cooperation with the European Respiratory Society (ERS) and the European Society of Thoracic Surgeons (ESTS)," Endoscopy, vol. 47, no. 6, pp. 545-559, 2015.

[2] C. R. Habermann, F. Weiss, R. Riecken et al., "Preoperative staging of gastric adenocarcinoma: comparison of helical CT and endoscopic US," Radiology, vol. 230, no. 2, pp. 465-471, 2004.

[3] T. de Potter, P. Flamen, E. van Cutsem et al., "Whole-body PET with FDG for the diagnosis of recurrent gastric cancer," European Journal of Nuclear Medicine and Molecular Imaging, vol. 29, no. 4, pp. 525-529, 2002.

[4] S. S. Larsen, P. Vilmann, K. Krasnik et al., "A comparison of endoscopic ultrasound guided biopsy and positron emission tomography with integrated computed tomography in lung cancer staging," Current Health Sciences Journal, vol. 35, no. 1, pp. 5-12, 2009.

[5] M. S. Bhutani, R. H. Hawes, and B. J. Hoffman, "A comparison of the accuracy of echo features during endoscopic ultrasound (EUS) and EUS-guided fine-needle aspiration for diagnosis of malignant lymph node invasion," Gastrointestinal Endoscopy, vol. 45, no. 6, pp. 474-479, 1997.

[6] A. Kanamori, Y. Hirooka, A. Itoh et al., "Usefulness of contrast-enhanced endoscopic ultrasonography in the differentiation between malignant and benign lymphadenopathy," The American Journal of Gastroenterology, vol. 101, no. 1, pp. 45-51, 2006.

[7] H. J. Song, J. O. Kim, S. H. Eun et al., "Endoscopic ultrasonograpic findings of benign mediastinal and abdominal lymphadenopathy confirmed by EUS-guided fine needle aspiration," Gut Liver., vol. 1, no. 1, pp. 068-073, 2007.

[8] P. Vilmann, G. K. Jacobsen, F. W. Henriksen, and S. Hancke, "Endoscopic ultrasonography with guided fine needle aspiration biopsy in pancreatic disease," Gastrointestinal Endoscopy, vol. 38, no. 2, pp. 172-173, 1992.

[9] I. Yasuda, H. Tsurumi, S. Omar et al., "Endoscopic ultrasoundguided fine-needle aspiration biopsy for lymphadenopathy of unknown origin," Endoscopy, vol. 38, no. 9, pp. 919-924, 2006.

[10] O. Nakahara, K. Yamao, V. Bhatia et al., "Usefulness of endoscopic ultrasound-guided fine needle aspiration (EUS-FNA) for undiagnosed intra-abdominal lymphadenopathy," Journal of Gastroenterology, vol. 44, no. 6, pp. 562-567, 2009.

[11] T. Iwashita, I. Yasuda, S. Doi et al., "Endoscopic ultrasoundguided fine-needle aspiration in patients with lymphadenopathy suspected of recurrent malignancy after curative treatment," Journal of Gastroenterology, vol. 44, no. 3, pp. 190196, 2009.

[12] R. Puri, R. Mangla, M. Eloubeidi, P. Vilmann, R. Thandassery, and R. Sud, "Diagnostic yield of EUS-guided FNA and cytology in suspected tubercular intra-abdominal lymphadenopathy," Gastrointestinal Endoscopy, vol. 75, no. 5, pp. 1005-1010, 2012.

[13] J. Korenblit, A. Anantharaman, D. E. Loren, T. E. Kowalski, and A. A. Siddiqui, "The role of endoscopic ultrasoundguided fine needle aspiration (eusfna) for the diagnosis of intra-abdominal lymphadenopathy of unknown origin," Journal of Interventional Gastroenterology, vol. 2, no. 4, pp. 172176, 2012.

[14] Y. N. Lee, J. H. Moon, H. K. Kim et al., "A triple approach for diagnostic assessment of endoscopic ultrasound-guided fine needle aspiration in pancreatic solid masses and lymph nodes," Digestive Diseases and Sciences, vol. 59, no. 9, pp. 2286-2293, 2014.

[15] Y. Tanisaka, S. Ryozawa, M. Kobayashi et al., "Usefulness of endoscopic ultrasound-guided fine needle aspiration for lymphadenopathy," Oncology Letters, vol. 15, no. 4, pp. 4759-4766, 2018.

[16] Y. Fujii, Y. Kanno, S. Koshita et al., "Predictive factors for inaccurate diagnosis of swollen lymph nodes in endoscopic ultrasound-guided fine needle aspiration," Clinical Endoscopy, vol. 52, no. 2, pp. 152-158, 2019.

[17] L. Chen, Y. Li, X. Gao et al., "High diagnostic accuracy and safety of endoscopic ultrasound-guided fine-needle aspiration in malignant lymph nodes: a systematic review and meta-analysis," Digestive Diseases and Sciences, 2020.

[18] D. G. Adler, B. Witt, B. Chadwick et al., "Pathologic evaluation of a new endoscopic ultrasound needle designed to obtain core 
tissue samples: a pilot study," Endoscopic Ultrasound, vol. 5, no. 3, pp. 178-183, 2016.

[19] J. Y. Bang, S. Hebert-Magee, M. K. Hasan, U. Navaneethan, R. Hawes, and S. Varadarajulu, "Endoscopic ultrasonography-guided biopsy using a Franseen needle design: initial assessment," Digestive Endoscopy, vol. 29, no. 3, pp. 338-346, 2017.

[20] M. A. Khan, I. S. Grimm, B. Ali et al., "A meta-analysis of endoscopic ultrasound-fine-needle aspiration compared to endoscopic ultrasound-fine-needle biopsy: diagnostic yield and the value of onsite cytopathological assessment," Endoscopy International Open, vol. 5, no. 5, pp. E363-E375, 2017.

[21] D. G. Adler, V. R. Muthusamy, D. S. Ehrlich et al., "A multicenter evaluation of a new EUS core biopsy needle: experience in 200 patients," Endoscopic Ultrasound, vol. 8, no. 2, pp. 99104, 2019.

[22] S. Mukai, T. Itoi, H. Yamaguchi et al., "A retrospective histological comparison of EUS-guided fine-needle biopsy using a novel franseen needle and a conventional end-cut type needle," Endoscopic Ultrasound, vol. 8, no. 1, pp. 50-57, 2019.

[23] M. Lin, C. D. Hair, L. K. Green et al., "Endoscopic ultrasoundguided fineneedle aspiration with on-site cytopathology versus core biopsy: a comparison of both techniques performed at the same endoscopic session," Endoscopy International Open, vol. 2, no. 4, pp. E220-E223, 2014.

[24] G. Mavrogenis, B. Weynand, A. Sibille et al., "25-gauge histology needle versus 22-gauge cytology needle in endoscopic ultrasonography-guided sampling of pancreatic lesions and lymphadenopathy," Endoscopy International Open, vol. 3, no. 1, pp. E63-E68, 2015.

[25] E. Rodrigues-Pinto, S. Jalaj, I. S. Grimm, and T. H. Baron, "Impact of EUS-guided fine-needle biopsy sampling with a new core needle on the need for onsite cytopathologic assessment: a preliminary study," Gastrointestinal Endoscopy, vol. 84, no. 6, pp. 1040-1046, 2016.

[26] J. Iglesias-Garcia, J. E. Dominguez-Munoz, I. Abdulkader et al., "Influence of on-site cytopathology evaluation on the diagnostic accuracy of endoscopic ultrasound-guided fine needle aspiration (EUS-FNA) of solid pancreatic masses," The American Journal of Gastroenterology, vol. 106, no. 9, pp. 1705-1710, 2011.

[27] S. Wani, V. R. Muthusamy, and S. Komanduri, "EUS-guided tissue acquisition: an evidence-based approach (with videos)," Gastrointestinal Endoscopy, vol. 80, no. 6, pp. 939-959.e7, 2014.

[28] S. F. Crinò, M. le Grazie, E. Manfrin et al., "Randomized trial comparing fork-tip and side-fenestrated needles for EUSguided fine-needle biopsy of solid pancreatic lesions," Gastrointestinal Endoscopy, vol. 92, no. 3, pp. 648-658.e2, 2020.

[29] Y. Nakai, H. Isayama, K. J. Chang et al., "Slow pull versus suction in endoscopic ultrasound-guided fine-needle aspiration of pancreatic solid masses," Digestive Diseases and Sciences, vol. 59, no. 7, pp. 1578-1585, 2014.

[30] P. Saxena, M. el Zein, T. Stevens et al., "Stylet slow-pull versus standard suction for endoscopic ultrasound-guided fineneedle aspiration of solid pancreatic lesions: a multicenter randomized trial," Endoscopy, vol. 50, no. 5, pp. 497-504, 2018.

[31] R. di Mitri, F. Mocciaro, F. Antonini et al., "Stylet slow-pull vs. standard suction technique for endoscopic ultrasound- guided fine needle biopsy in pancreatic solid lesions using 20 Gauge Procore ${ }^{\mathrm{TM}}$ needle: A multicenter randomized trial," Digestive and Liver Disease, vol. 52, no. 2, pp. 178-184, 2020. 